\title{
THREE DIMENSIONAL RUPTURE ANALYSIS OF A PRESTRESSED CONCRETE PRESSURE VESSEL INCLUDING CREEP EFFECTS
}

\author{
B. SAUGY, Th. ZIMMERMANN, M. HUSSAIN KHAN \\ Institut de production d'énergie, Département du génie civil, \\ Ecole Polytechnique fédérale de Lausanne, CH-1006 Lausanne, Switzerland
}

\section{SUMMARY}

This paper describes briefly the results obtained since September 1971 by the research work carried out in our Institute in the field of non-linear analysis up to rupture of a PCRV taking into account of creep effects.

In the earlier work done in this field (and reported in paper H3/5, Vol. 4, 231-251 presented at the 1st SMIRT Conference) the influence of redistribution of stresses was restricted to short-time effects in the evaluation of rupture pressure of a PCRV. The research carried out since then aimed firstly to make a synthesis of constitutive laws describing the behavior of concrete under short-time or sustained loads and temperature gradients and of its rupture strength under tri-axial state of stresses which allowed to bring into evidence the principal parameters governing the behavior of concrete and to take them into account in the analysis. Secondly, it aimed to examine the influence of redistribution of stresses due to the rheological behavior of concrete on the rupture pressure of a PCRV.

First the method of non-linear analysis for creep and rupture is described briefly. The mathematical model, of a general application, is based on the Finite Element Method, utilising the isoparametric elements, non-linearities are introduced by use of iterative techniques. It allows to predict under satisfactory limit the behavior of massive prestressed concrete structures loaded up to destruction. The use of classical parameters for definition of the physical characteristics of materials for formulating the constitutive laws makes the model particularly interesting for practical applications.

Then the analysis of the PCRV for a Gas-Cooled Fast Reactor developed by the Swiss Federal Institute for Reactor Research is presented. This PCRV has large cavities in its walls to house direct cycle gas turbines and other mechanical equipments. First the creep analysis is done taking account of the envisaged construction schedule and the loading conditions of internal pressure and thermal gradients created during the testing period and finally during the normal exploitation of the PCRV. Proceeding from the state of stress obtained as such the rupture analysis is carried out for a sudden increase of internal pressure and of temperature gradient due to an hypothetical accident. 
1.

\section{Introduction}

The work described in this paper is carried out under the direction of Professor Dr A. Gardel at the Swiss Federal Institute of Technology in Lausanne and financed by the Swiss National Fund for Scientific Research. This theoretical research aims to develop deformation models predicting the non-linear behavior of massive structures. In a first phase, a deformation model to predict short time load effects was developed and a computer code for three dimensional analysis based on the Finite Element Method was established. This model gave satisfactory results for analysis of structures such as end slabs of a cylindrical PCRV, tunnels in rock massifs and foundations. The following phase aims to define a conventional model of creep which integrates suitably in the first model and which will allow to analyse the behavior of the structure upto rupture under increasing loads taking into account the evolution of material properties and the state of stress and strain in function of time. This model is being tested to analyse structu res submitted to laboratory tests. Besides these tests it appeared interesting to apply it to a real structure for which such an analysis seems to be necessary. The PCRV for a Gas-Cooled Fast Reactor of 1000 MWe developped by the Swiss Federal Institute for Reactor Research (EIR) has been retained for such an analysis. We are thankful to EIR and in particular to Dr Sarlos for furnishing us their repart on the assessment studies of this reactor.

\section{Description of the PCRV and of the Loads}

The integrated variant of the Helium-Cooled Fast Reactor of 1000 MWe capacity retained by the EIR is a three loop design. Beside the reactor core, three helium driven turbosets, each composed of two compressors and two turbines capable of generating 335 MWe, and six heat recuperators (two per turboset) are housed in the PCRV. Three electrical generators are vertically arranged under the PCRV.

The PCRV is a cylindrical type vessel of $29,5 \mathrm{~m}$. external diameter (Fig. 1). The reactor core is housed in a central cylindrical cavity of $6,7 \mathrm{~m}$. diameter and $13,3 \mathrm{~m}$. height. Nine large vertical cylindrical cavities, each of 3,9 $\mathrm{m}$. diameter and of $33,9 \mathrm{~m}$. height are provided in the vessel wall and arranged in a symmetrical manner around the reactor cavity. The turbosets and the recuperators are housed in them. The PCRV has a reduced height of $27,7 \mathrm{~m}$. In the central part of $9,5 \mathrm{~m}$. diameter and a height of $36,9 \mathrm{~m}$. for the rest of the structure. Three penetrations of different diameters, varying from $81 \mathrm{~cm}$. to $157 \mathrm{~cm}$., connect each of the recuperator cavities with the turboset and reactor core cavities. Each turboset cavity is connected to the reactor cavity by a horizontal penetration of $73 \mathrm{~cm}$. diameter.

The gas pressures and temperatures for the working conditions of the power station are as described in table $I$.

For more details on flow and temperature-entropy diagrams of the direct HeliumTurbine cycle the reader may refer to the EIR report [1].

The loading conditions taken into account in tho prescnt analysis are shown in the 1 igure 2. The origine of time in the diagrams shown in this figure is taken as the date of realization of $\mathrm{s}(\mathrm{l}) \%$ of the concreting of the P'ikV. Aftur one year from this date 
$100 \%$ of the prestressing loads are applied. At the age of 3 years, the loads corresponding to the normal working conditions of the power station are applied. These loads are admitted to be the following :

- internal pressure of 90,9 bars in the reactor cavity,

- average internal pressure of 35,7 bars in the peripheral cavities,

- internal temperature of $50^{\circ} \mathrm{C}$ in the concrete adjacent to the liner of all the cavities and temperature of $20^{\circ} \mathrm{C}$ on all the external walls of the PCRV.

The maximum hypothetical accident is supposed to occur after 2 years of normal servicing of the power station. At this moment the internal pressure in the peripheral cavities suddenly increases to 90,9 bars and then the pressure in all the cavities cantinues to increase simultaneously till the rupture of the pressure vessel occurs. Unsymmetrical loading conditions as well as proof test, start up, shut down and faulty operational condition of the liner cooling water system have not been examined in the present study.

3.

\section{Design criteria}

The design criteria can be grouped under four following headings.

a) Limiting allowable state of stress for construction and normal operating conditions with sustained loads (long time behavior) including start up and shut down loadings.

b) Limiting allowable state of deformation for normal operating conditions with sustained loads.

c) Limiting allowable state of stress for short-time exceptional operating and faulty functionning conditions.

d) Minimun ultimate load capacity.

If $\beta_{w}$ is the cube strength of concrete at 28 days, the group a) criterta limits the maximum stresses to 0,33 to $0,4 B_{w}$ in compression and 0 to $10 \mathrm{~kg} / \mathrm{cm}^{2}$ in tension. In the zones where there are stress concentratians in general, around penetrations in particular, these limiting values are increased upto 1,5 to 2 times as the penetration liner and the reinforcement around it are not taken into account in the analysis.

The group b] criteria limits the maximum values of deformations and displacements which are imposed by the liner designer so that the amount of strain cycling to which liner is submitted remains in acceptable limits and by the equipment designer, in particular of the control-rod assembly. The deformations are limited to 0,5 to 0,8 o/oo and the displacements to 2 to $3 \mathrm{~mm}$.

The group c) criteria limits the stress to 0,45 to $0,5 \beta_{w}$ in compression and to 10 to $30 \mathrm{~kg} / \mathrm{cm}^{2}$ in tension when there is a $20 \%$ rise in internal pressure and the temperature gradient is increased by $50 \%$ due to a faulty functionning of the liner cooling water system.

Finally the minimum ultimate load capacity for a sudden rise in pressure simultaneously in all the cavities is required to be of the order of 3 times the pressure in the reactor cavity during normal operating condition. 
4. Constitutive laws

\subsection{Stress-strain law}

An isotropic non-homogeneous model with charactaristics varying in function of the state of stress, of the temperature and of the loading age of the material has been used. The constitutive laws are expressed in Lame's tensorial form for the initial elastic model.

$$
\begin{aligned}
S & =2 \mu_{B} \\
\sigma_{m} & =3 K E_{m} \\
\text { where } S & =\text { deviatoric stress } \\
e & =\text { distorsion } \\
\mu & =\text { shear modulus } \\
K & =\text { bulk modulus } \\
\sigma_{m} & =\text { average stress } \\
\epsilon_{m} & =\text { average strain }
\end{aligned}
$$

The non-linear behavior of concrete under statical short-time loads is 1dealised in the follawing manner.

$$
\begin{array}{ll}
K=\text { canstant } \\
\mu=\mu_{e}-\alpha \log _{e}\left(I_{2} / I_{2 e}\right) \\
\mu=\mu_{e} \text { for } I_{2}>I_{2 e} \\
\text { for } I_{2} \leqslant I_{2 e}
\end{array}
$$

where $I_{2}=1,5 \Sigma S_{1}^{2}$ is the second invariant of the stress tensor and the Index $e$ ind1cates elastic behavior range. These laws have been established on the bas1s of experimental results published by several authors and commented in an earlier paper [2]. They have already been successfully tested and campared with published experlmental studieg.

The nan-linear model presented above is very practical as far as application $1 \mathrm{~s}$ concerned as it usesexclusively current material characteristics. The proposed rheological model which follows does not require also any further special experiments and adapts naturally to the non-linear one. This rheologlcal model which defines the secant shear and bulk moduli in function of time 1 s of the form :

$$
\begin{aligned}
& \mu_{(t-\tau)}=\mu_{i} /\left(1+a(t-\tau)^{n}\right) \\
& k_{(t-\tau)}=k_{i} /\left(1+a(t-\tau)^{n}\right) \\
& \text { where } \tau=\text { loading age } \\
& t \quad=\text { time } \\
& a \text { and } n \text { are creep factors }
\end{aligned}
$$

and index 1 designates the value at loading age.

The evolution of the values of $\mu$ and $K$ in function of $\tau$ et $t$ admitted in the present analysis is shown 1n the Figure 3. Firstly the instantaneous shear modulus

is determined in fonction of the second Invariant of the stress tensor $I_{2}$ with the aid of Figure 3a). Then the effect of age 1s taken into account with the ald of Figure 3b) which allows to determine the value of $\mu_{1}$ at the time $\tau$ of the application of the 
loads. The figure 3cl gives the evolution of the value of the creep factor a in function of the loading age $\tau$ and the temperature $T$ of the concrete. The value of a is independent of the state of stress. Even for a good degree of precision, the creep factor $n$ can be assumed constant. Finally the Flgure $3 d$ ) allows to determine the fictive secant modulus $\mu_{(t-\tau)}$ in function of the parameter $a \cdot(t-\tau)^{n}$ which itself is evaluated from the known values of the creep factors and $(t-\tau)$ the time under load. The value of $k$ ( $t-\tau)$ is determined in a similar manner.

It may be remarked that the equation (3) applied to a one dimensional case corresponds to a creep law, defining the deformation, of the following type :

$$
E=E_{i}\left\{1+a(t-\tau)^{n}\right\}
$$

\subsection{Rupture criterium}

This criterium had been discussed in a more detailed form in an earlier paper [4]. We will just recall here its essential elements. The range of validity of the laws described in the previous paragraph is limited by a rupture surface $\psi$ in the space of principal stresses of the type:

$$
\psi=f\left(\sigma_{1}, \sigma_{2}, \sigma_{3}\right)
$$

and defined in a speolfic manner in terms of the stress invariants by the following equations :

$$
\begin{aligned}
I_{1} & =\sum_{1=0}^{3} \alpha_{i}\left(I_{2}^{0,5}-E\right)^{i} \\
\text { with } E & =\alpha_{4}-\alpha_{4} /\left\{\left(I_{2}^{0,5}-E\right)^{2} T^{2} /\left(\alpha_{4}+\gamma / 2\right)+\left(I_{2}^{0,5}-E\right) T+\alpha_{4}\right\} \\
T & =\left\{1 / \cos \left(\Sigma 1,5\left(S_{1} / I_{2}^{0,5}\right)^{3}\right\}-1\right.
\end{aligned}
$$

where the $I_{1}$ are the stress invariants; the $\alpha_{i}$ are functions of $\beta_{c}$. $m$ and $\gamma$. The uniaxlal compressive strength on cyl. $\beta_{c}$ and the coefficients $m$, the ratio of biaxlal to uniaxial compressive strength, and $\gamma$, the ratio of uniaxial tensile to compressive strength define completely the surface of rupture. The five parameters $\alpha_{0}$ to $\alpha_{4}$ are determined by utilizing the five points corresponding to the fallowing states of stresses :

Defintion of the state of stress

unlaxial compression
biaxlal compression
unlaxial tension
biaxial tension
triaxial tension

Principal stresses

$$
\begin{array}{lll}
\sigma_{1} & \sigma_{2} & \sigma_{3}
\end{array}
$$$$
\beta_{\mathrm{c}} 0 \quad 0
$$$$
m \beta_{c} m \beta_{c} \quad
$$$$
\begin{array}{lll}
Y B_{C} & 0
\end{array}
$$$$
\gamma \beta_{c} \gamma \beta_{c} 0
$$$$
\gamma \beta_{C} \quad \gamma \beta_{C} \quad \gamma \beta_{C}
$$

The parameters $B_{c}, m$ and $Y$ vary $1 n$ function of the age of concrete. As such the general equation of the surface of rupture $1 \mathrm{~s}$ of the type

$$
\psi(\sigma)=\psi\left(\beta_{c}, m, \gamma, \tau\right)
$$


The rupture defined by such a surface depends on the average pressure, the deviatoric stress, the ratio of principal stresses and the age of the concrete.

The tensile cracking of the concrete is taken into account by introducing a local orthotrapy oriented along the plane of cracking and placed discontinuously in the model at the integration points of the finite elements. The stresses incompatible with the local orthotropy are then corrected and redistributed in the structure through fictive equivalent nodal forces.

5 .

Mathematical model

The basic numerical model utilizes the elastic isoparametric elements with 20 nodal points elaborated at the University of Swansea by the research group of Professor a.C. Zienkiewicz [5]. A frontal elimination process developped by B. Irons [6] is used in the computer cade established to solve the set or linear equations.

The non linear behavior of the material is taken into account by a cycle of itera tions of the pseuda-elastic model without modifying the stiffness matrix. At each iteration, fictive loads, corresponding to the difference between pseudo-elastic model and the constitutive laws idealizing the non-linear and sheological behavior of the material, are introduced as external forces at the nodal points. In fact these fictive forces are the unbalanced forces which the material can not take up in the zone considered. The salution obtained at the end of the cycle of iteration is the one which simultaneously satisfies with satisfactory precision the internal equilibrium and the canstitutive laws at a given number of integration points of each element.

The computing time which tends to become excessive for such an iteration process has been fairly reduced by the introduction of accelerating processes based on the interpolation of values of the variables abtained during the preceeding iterations and optimized in the structure by a least square process.

The principle of this iterative process is shown in the figure 4 which is valid for symbollc tensorial stress created flrstly by an increasing or a decreasing loading candition realized at constant velocity and secondly by creep effects. The non-linear constitutive law is sat1sfied by defining the triangle ABC in which oj corresponds to the unbalanced stress which is equal to the difference between the elastic model and the non-linear one. When the equilibrium is obtained with a sufficient precision at each point, the real deformation is equal to $\varepsilon_{e}$ plus $\varepsilon_{n l}$ The evolution in function of time is then searched by a new cycle of iterations in which the stress $\sigma_{c}$, equivalent to the creep defarmation defined by the corresponding constitutive law, is evaluated at each step and 1ntroduced as a fictive stress. If creep leads to an accelaration of the fallure process, a new non-linear equilibrium is searched out according to the first type of iterative ргоcess.

6

Analysis

The introduction of rheological model in the computer code 1 s actually at the testing atage. We therefore choose a rather simple finite slement mesh for the present analy- 
sis to verify, the algorithms and to avoid prohibitive computer time.

The PCRV has a symmetry of the order of 18 as it has 9 cylindrical peripheral cavities. The Figure 6 shows the fintte element mesh for 1/18th part of the PCRV. It comprises of 04 isoparametric elements and 805 nodal points.

For normal operation conditions the prestressing tendons are not intraduced in the mesh and uniform equivalent pressures representative of the prestressing forces are applied. The state of stress and deformation is determined for internal pressures in the cavity, thermal gradient and the prestressing forces and 1 t is checked that the stress and deformation limits mentionned earlier (paragraph 3) are satisfled and that there is reversibllity of deformations at all parts of the structure.

While examining the behavior of the structure under increasing pressure loads upto rupture, one dimensional steel elements are introduced in the finite element mesh to simulate the prestressing tendons so that a better picture of the failure mechanism is obtained.

First a creep analysis is done taking into account of the history of loading, then the pressure in the cavities is increased by steps till failure of the structure is obta1ned. Till now an analysis with complete loading and unloading cycle has not been attempted on the present model as it will be time consuming and expensive.

The introduction of steel elements in the finite element mesh for the analysis of composite structures like the PCRV allows to take into account of the redistribution of stresses, in a more realistic manner, between the concrete and the steel. Th1s redistribution influences the ultimate load capac1ty of the structure to a degree depending upon the quantity and the location of the steel members.

Actually only test analysis have been carried out by now to check the algorithms of the mathematical model. Final results have not been obtalned and therefore can not be given now. Moreover, the influence of the varlation of some principal parameters of the constitutive laws on the behavior of the PCRV 1 s also under study actually. All these results wili be presented at the 2nd SMIRT conference and published later. 
EIR, "1000 MWe Hellum Cooled Fast Reactor, Assessment studies", Sw1ss Federal Institute for Reactor Research, Report no TM-St-260, Würenlingen, July 1972.

SAUGY, B., "Contribution à l'étude théorique du comportement des structures massives en béton armé sous charges rapides", Bulletin technique de la Suisse romande, по 22, 1969.

FREUDENTHAL, A.M., ROLL, F., "Creep and Creep Recovery of Concrete under High Compressive Stress", Journal of the American Concrete Institute, no 12, June 1958.

HUSSAIN, M., SAUGY, B., ZIMMERMANN, Th., "Analyse tridimensionnel le du comportement non linéaire d'un caisson de réacteur nucléaire en béton précontraint", Proceedings of the 1st SMIRT Conference, Berlin 1971.

ERgatounis, J., "Isoparametric Finite Element in two and three Dimensional Stress Analysis ", Ph. D. Thesis, University of Wales, Swansea 1968.

IRONS, B., "A Frontal solution Program for Finite Element Analysis", Int. J. for Numerical Methods in Engineering, по 1, Vol, 2, 1970.

Table I. Normal operational condition pressure and temperature characteristics

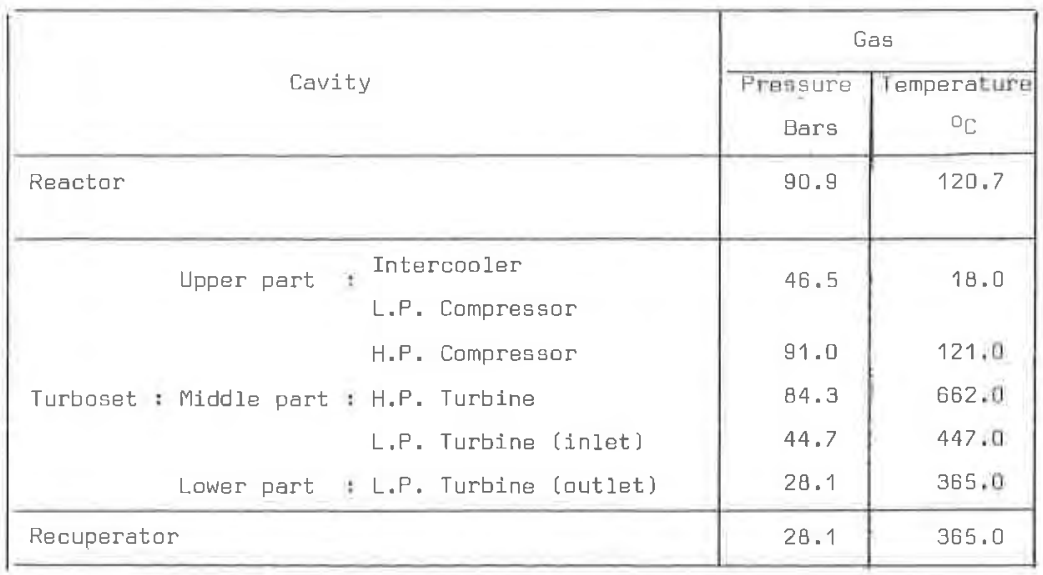




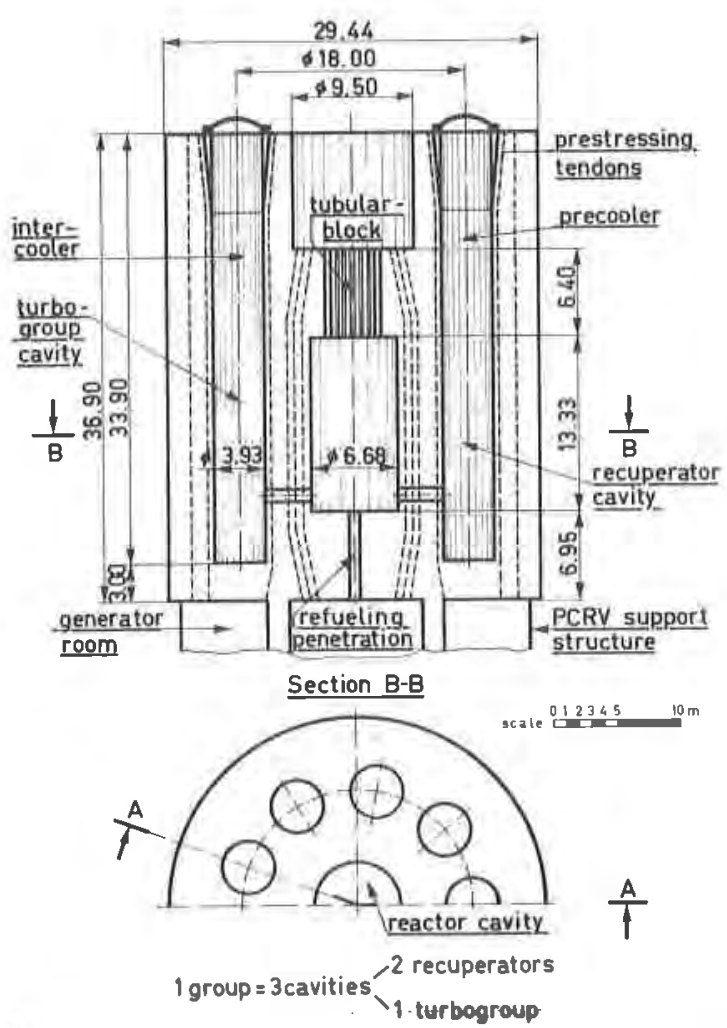

1. PCRV of a 1000 MWe helium cooled fast breeder with direct cycle gas turbines.
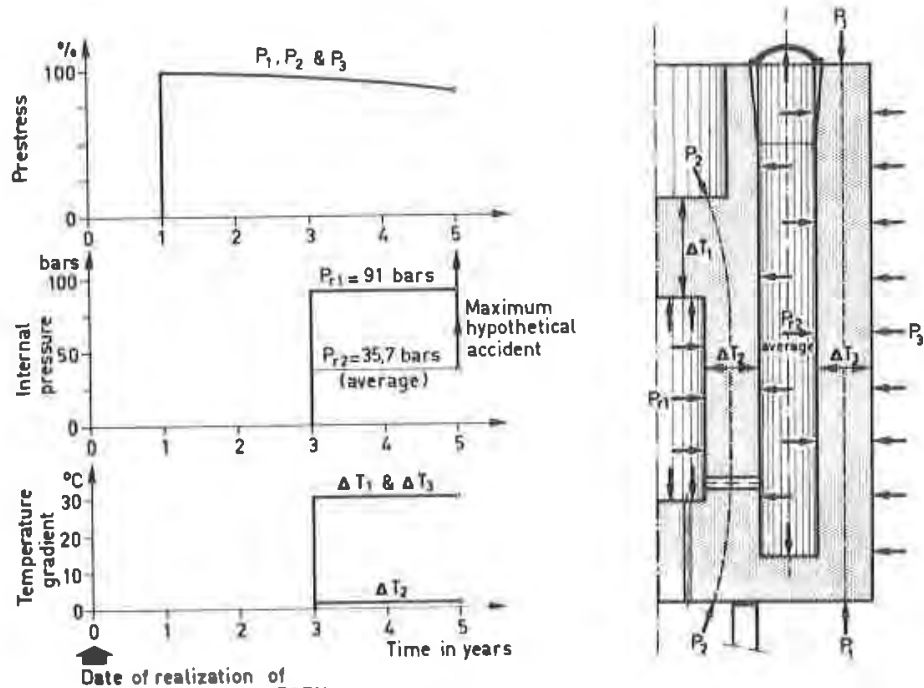

$50 \%$ of concrete of PCRV

$\%$ Varlation of loads and temporature gradlont in functlur of $t 1 \mathrm{mr}$. 


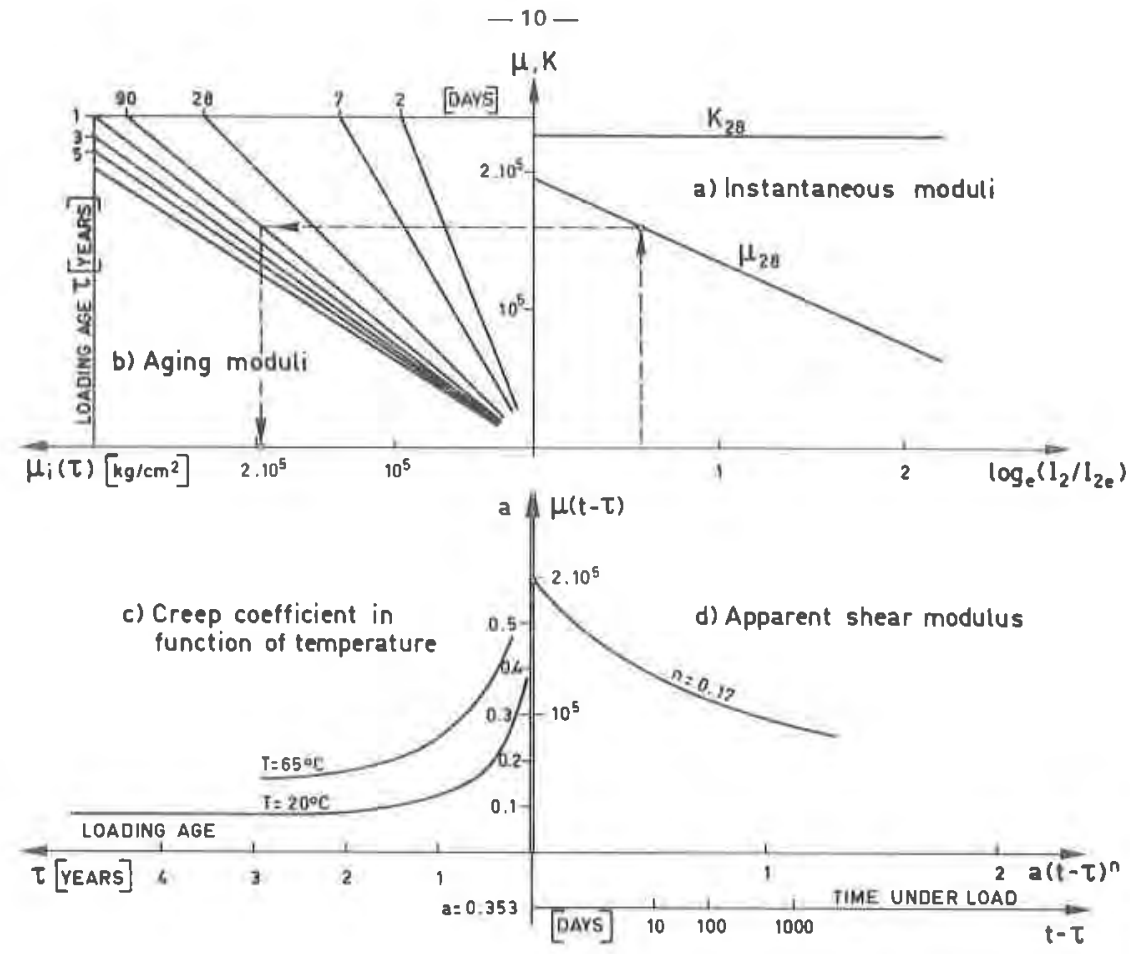

3. Constitutive laws,

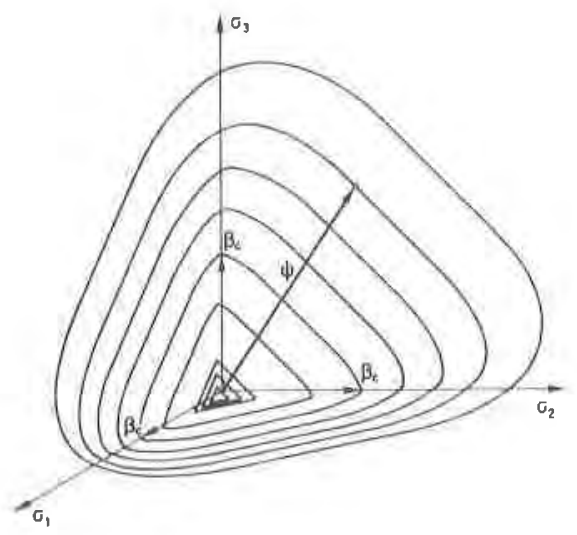

$\psi\left(\sigma_{1}, \sigma_{2}, \sigma_{3}\right)=\psi\left(\beta_{c}, m, n, \tau\right)$

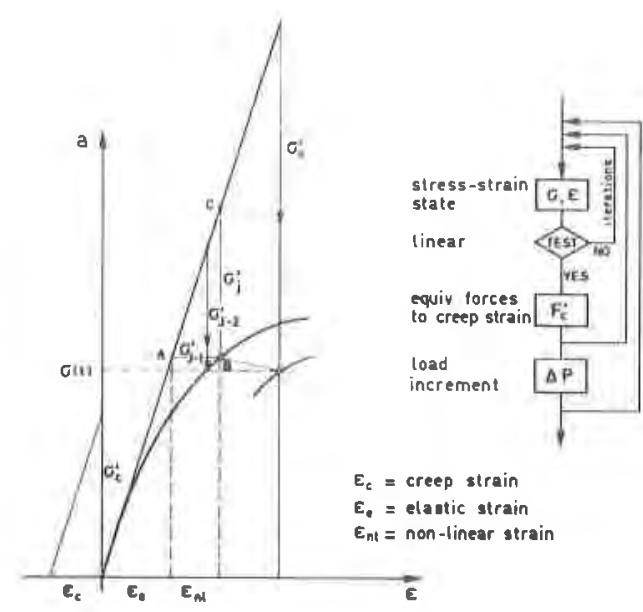

5. Rupture criterium. 
84 Elements 805 Nodes

6. Finite element mesh.

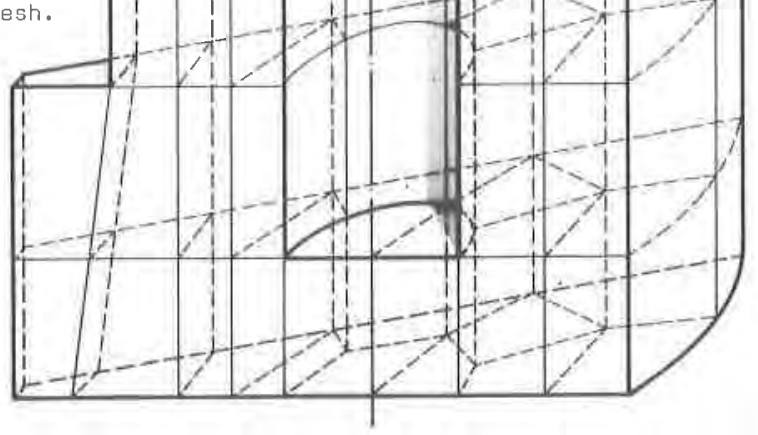


